\title{
Comparative Analysis of Feature Extraction and Pixel-based Classification of High-Resolution Satellite Images Using Geospatial Techniques
}

\author{
Alaa Adnan Musleh ${ }^{1, a^{*}}$ and Hussein Sabah Jaber ${ }^{1, b}$ \\ ${ }^{1}$ Surveying Engineering Department, University of Baghdad, Baghdad, Iraq \\ aalaa.adnan2014@gmail.com, bhusseinsabah00@gmail.com
}

\begin{abstract}
Two common techniques for classifying satellite imagery are pixel-based and Feature extraction image analysis methods. Typically, for agreements reached imaging, pixel-based analysis is used, whereas high-resolution imagery is suitable for Feature extraction analysis. However, In the classification of moderate images, image segmentation's ability depending on criteria such as shape, color, texture, and spatial features in Feature extraction image analysis implies it can perform better than pixel-based analysis. A comparative study of the two methods was performed using Sentinel- 2 imagery from 18 May 2020 to categorize LU/LC in the City of Baghdad, Iraq. After calculating LU/LC for Baghdad images' capital, a supervised classification was performed using the two methods. The images used have been the support vector machines (SVM) and the maximum likelihood classification (MLC) for pixel-based method and Feature extraction method, which is available in ENVI and ArcGIS software packages, respectively. Land cover and land use classes included five Groups (vegetation area, asphalt road, soil area, water body, and built-up) was found that the Feature extraction methodology produced higher overall accuracy and Kappa index in the city of Baghdad image. The highest achieved accuracy for the Feature extraction technique was overall accuracy $95 \%$ with Kappa index 0.94 of SVM and overall accuracy of 92\% with Kappa index 0.90 of MLC. In comparison, the highest accuracy for the pixel-based was overall accuracy $88 \%$ with Kappa index 0.84 of SVM and overall accuracy $86 \%$ with Kappa index 0.82 of MLC.
\end{abstract}

Keywords: ENVI; land use; land cover; remote sensing; pixel-based; feature extraction; GIS.

\section{Introduction}

In supplying and producing geospatial data, satellite images play a vital role. Qualitative and quantitative information is given by satellite and remote sensing images that reduce fieldwork and study time complexity. Remote sensing data technologies gather images/data during steady intervals. The massive amount of data collected in data centers is immense and continuously increasing as the technology is increasingly evolving as timely data amounts have risen exponentially [1]. For the extraction and analysis of useful information based on enormous satellite images, effective and efficient mechanisms are extremely necessary. Classification of the satellite image is considered a robust technique to collect data from many satellite images. There is a clear need for a similar comparison of the feature extraction and pixel-based classification of high-resolution satellite images using remote sensing, with many aspects relevant to general fieldwork. It is also be referred to as satellite image classification as extracting data from satellite images. Satellite image classification is not very difficult, but in the processing of satellite images, the observer must make multiple decisions and choices. Satellite image classification involves interpreting remote sensing images and spatial data mining, studying the different classification types described in this research $[1,2]$.

This study will focus on multispectral satellite images from the sentinel satellites to compare two types of classification. The European Space Agency launched the Sentinel-2A satellite in June 2015 and then followed by the Sentinel-2B in March 2017. The satellites take images with the repeated cycle in $786 \mathrm{~km}$ height, Ten days on a single satellite, and five days on two satellites. The goal is to provide information and contribution to land observations, emergency response and security services, agricultural practices, and forestry and assist in food management [2]. 


\section{Study Area}

Baghdad city is the study area. It is the capital and Iraq's largest administrative base. Baghdad is situated on both sides of the Tigris River in the central part of Iraq, with geographical coordinates: Longitude $\left(44^{\circ} 12^{\prime} \mathrm{E}\right)$ to $\left(44^{\circ} 33^{\prime} \mathrm{E}\right)$ and Latitude $\left(33^{\circ} 9^{\prime} \mathrm{N}\right)$ to $\left(33^{\circ} 30^{\prime} \mathrm{N}\right)$. In Iraq, Baghdad is the largest and most densely populated city. The city is crossed by the Tigris River and split into two groups: Karkh (west coast) and Rusaffa (East coast). From the east, the region is bordered by the River Diyala, which joins the River Tigris southeast of Baghdad, as shown in Figure 1 [3].

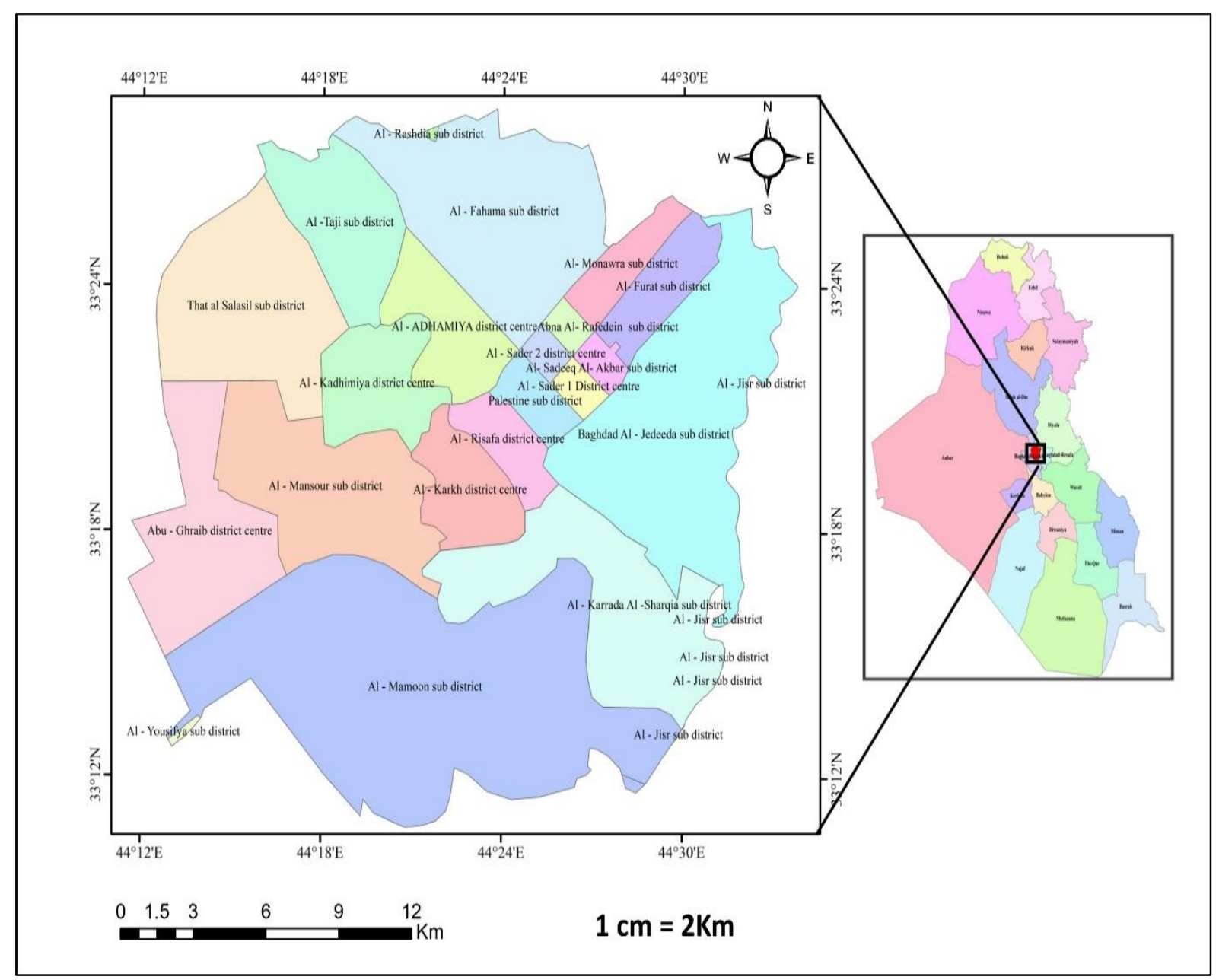

Figure 1. Map of the study area.

\section{Theoretical Framework}

Feature Extraction Classification. Feature extraction classification is distinguished from the pixelbased classification method because, instead of direct pixels, it operates on the pixel group. Feature extraction classification has two steps: (1) segmentation of images for producing segmented images, and (2) segmented image classification [4]. The essential and crucial step in Feature extraction classification is image segmentation. Classification efficiency is influenced by the effects of the process of image segmentation. The segmentation of images separates an image into objects. However, the bottom-up principle is often used in Feature extraction image analysis since it contrasts all the characteristics of small objects to obtain larger objects after merging. Under the Bottom-up theory, several segmentation techniques are available, but the increasing segmentation method of the multi-resolution region is typically used in remote sensing image analysis.

The fusion of two adjacent segments meets specific homogeneity requirements based on size, spectral and spatial information parameters. The threshold setting that can trigger over or under segmentation is the main problem of regional development and may not build desired objects for 
different targets. No segmentation findings can be proven if the human eye is not fulfilled $[4,5]$. The enormous bandwidth approached by segmentation applications that have already been outlined here will definitely lead to more progress required to use the full potential of remotely sensed information. Spectrum character, geometry, shape, size, and structure information are included in the image analysis. Feature extraction approaches to image analysis aim to solve the limitations that exist in PBC by grouping pixels into higher-level objects. The Feature extraction classification can be accomplished using the very reliable Definiens ENVI 5.3 program, which also requires a segmentation stage before classification.

Pixel-Based Classification. As a conventional method of remote sensing image classification, the pixel-based classification approaches assume that each of the pixels is pure, also majorly labeled as the type of the single land utilization land cover. In such an approach, the remote sensing imagery has been specified as a set of pixels with spectral information. Thus the spectral variables and their transformation (for instance, vegetation indices, principal components, and so on) have been input to per-pixel classifiers. Generally, the pixel-based methods of classification might be categorized into two classes: supervised and unsupervised classifications. Concerning the latter, remote sensing image has been split into several categories based on natural groupings related to the image's values, with no help of the training data or previous knowledge of the research field. There are two un-supervised classification algorithms, k-means, in addition to its variants, the Iterative Self-Organizing Data Analysis (ISODATA) approach, which has been the majorly utilized classifiers. Today, the SelfOrganizing Maps (SOM) approach and the hierarchical clustering approach have been designed for unsupervised classification $[5,6]$.

In contrast, with the supervised classifiers, the image analyst in selecting representative sample sites with the known types of the classes (for instance, the samples of the training). Also comparing spectral characteristics regarding every one of the pixels in the image with the ones of the training samples, labeling the pixels to the class type based on decision rules. Many supervised classification techniques were designed, such as MLC, k-NN classifier, Parallel-piped, Mahalanobis Distance Classifier, and Minimum Distance Classifier. Now, machine learning approaches were designed for refining the knowledge learning process; also, such approaches involve ANN, SVM, and classification trees [5].

Maximum-likelihood classification (MLC). One of the most widely used classifiers to date is the maximum probability classifier, a multivariate classifier that suggests each class is uniformly distributed across all bands. Depending on the likelihood of belonging to a specific class, every pixel is assigned to a class. With training samples, the algorithm is trained, generating a number of classes and an $\mathrm{N}$ number of ellipsoid probability functions. In contrast, variability or correlation indexes are computed [7]. Not all pixels fall perfectly in these functions of chance but still within the periphery. One can avoid pixels being crudely assigned to a class with a very low probability by adding a probability threshold number $[7,8]$. However, it would remain unclassified if the pixel did not satisfy this threshold Equation (1).

$$
\text { Dis }=\ln \left(\mathbf{b}_{\mathbf{i}}\right)-\left[0.5 \ln \left(\left|\operatorname{Cov}_{\mathbf{i}}\right|\right)\right]-\left[0.5\left(\mathrm{Y}-\mathbf{N}_{\mathbf{i}}\right)^{\mathrm{T}}\left(\operatorname{Cov}_{\mathbf{i}}^{-1}\right)\left(\mathrm{Y}-\mathrm{N}_{\mathbf{i}}\right)\right]
$$

Where (Dis) is the weighted distance, (bi) is a unique class, (Y) is the statistical signature vector of the test pixel, (Ni) is the mean sample vector of the class (bi) (which is believed to be equal probability), (Covi) is the modulation factor of the covariance matrix of the training data belonging to class (bi), and T. This class, which has the greatest probability (Dis), will be allocated the pixel.

Support Vector Machine classification (SVM). In remote sensing applications, SVM has been broadly adopted for imagery classification. To find an optimal boundary between groups, the SVM aims to achieve the optimal separation of a hyperplane and hyperplane in high and dimensional space. This method's methodology was proposed by the researchers Vapnik and Chervonenkis and later discussed by the author Vapnik [8]. The help vector machine offers both a learning model for classification and a mathematical algorithm. To allow linear domain division, with a model of $y=$ 
$\mathrm{wx}^{\prime}+\Upsilon$ that also manipulates it. Help vector machines are structures that are either linear or nonlinear. If linear, the data domain can be divided to break the classes linearly $[2,8]$. If the data has to be converted into a feature space because it is nonlinear, the data can then be divided linearly. This is achieved by using a polynomial kernel to construct a new space [8].

\section{Methodology}

This research methodology focuses on using accurate, modern, and cost-effective methods compared to traditional high-cost methods for producing thematic maps LU/LC, showing the difference between supervised classification methods (Feature extraction and pixel-based), clarifying which is better. The schematic of the research methodology is shown in Figure 2.

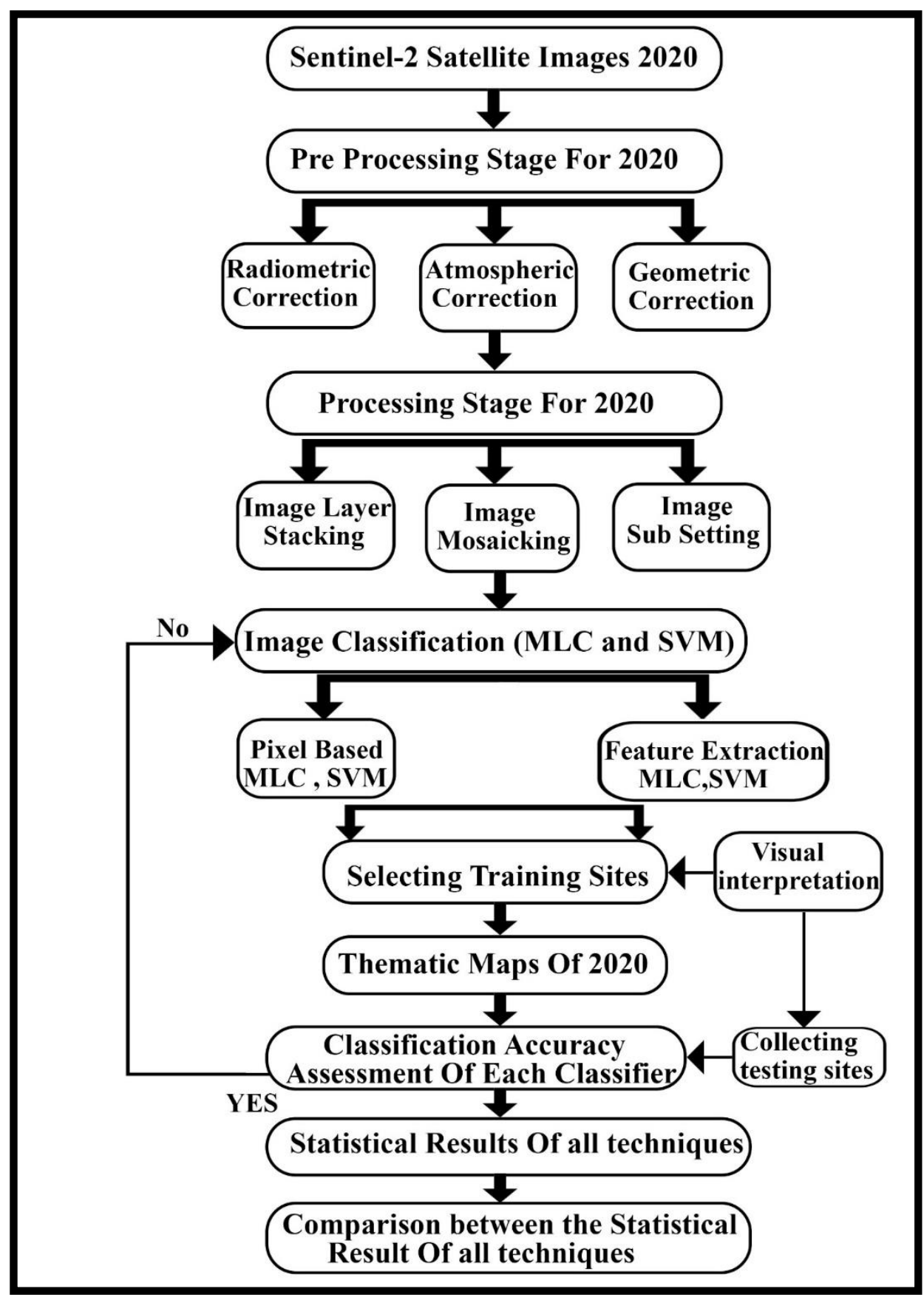

Figure 2. The schematic of research methodology.

Satellite Imagery Acquisition. In our work, for its low cost, particularly concerning the direction covered, Sentinel satellite images have been used. Another advantage of the Sentinel image is 
ownership, enabling the federal agency to exchange information legally, academia and donor agency. Furthermore, during work, two satellite images were analyzed; Sentinel 2 satellite image (28/5/2020). The images used in this work were taken during the same season (summer season), were not highly affected by the atmosphere (scattering and absorption), and were cloud-free. Satellite images used for this work have been collected from USGS Earth Explorer's database ("http://earthexplorer.usgs.gov/") as shown in Figure 3.

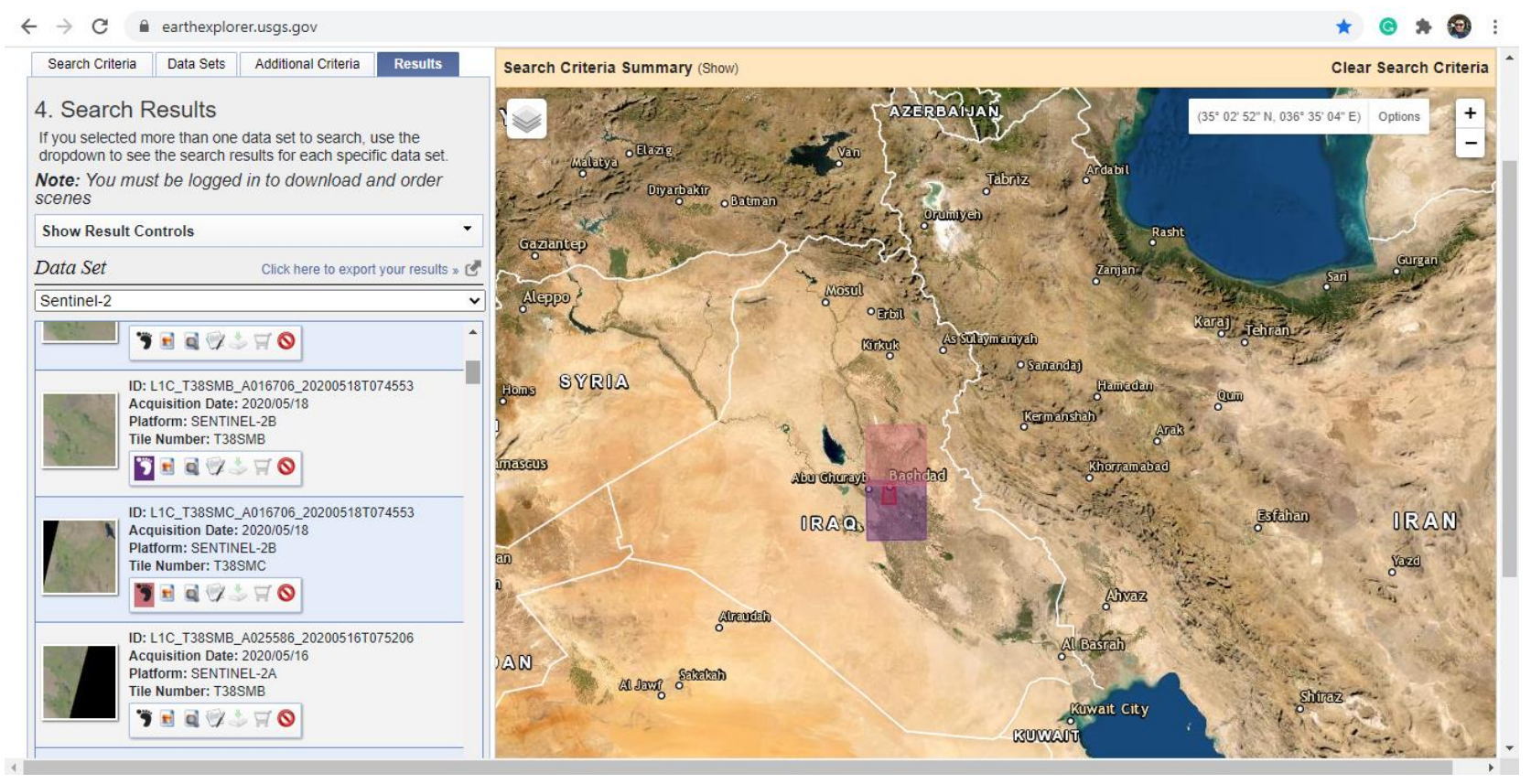

Figure 3. The website of USGS https://earthexplorer.usgs.gov/.

Pre Processing of Satellite Images. In general, pure satellite images contain inevitable mistakes and will not be explicitly used to identify features and applications [7, 9]. It requires specific rectification. Until the primary data analysis and data retrieval, pre-processing is completed. Two significant procedures include pre-processing, Geometric correction, and cloud correction or radiometric correction [9]. ERDAS 14 software [10] was used in this paper to perform most pre-processing phases of a satellite image.

Geometric Correction of Satellite Images. Geometric data correction is a crucial step for the classification of images [10]. 7GCPs gathered from fieldwork to conduct the geometric correction and select an appropriate projection for the image utilized. All satellite images collected from the site (http://earthexplorer.usgs.gov/) were geo-registered with the same Universal Transversal Mercator "(WGS 1984 UTM Zone 38 N)" coordinate [11]. Subsequently, all satellite images (Sentinel 2) Conducted out along with WGS84 data and UTM Zone $38 \mathrm{~N}$ prediction, using the nearest neighbor's re-sampling, as shown in Figure 4.

Atmospheric and Radiometric Correction of Satellite Images. Since digital sensors register the intensity of electromagnetic radiation as a digital number within each spectral band to every spot viewed on the Surface of the earth, the same DN range that a sensor used relies on its radiometric resolution $[11,12]$. Therefore, it is strictly essential to normalize image pixel values for variations in sun radiation geometry. The atmospheric effects and instrument calibration. The radiometric correction was used to restore the image using sensor calibration to ensure the output uniformity across the image face and over time [12]. The ERDAS 14 program was used with "Radiometric Correction Tools" to perform radiometric correction and atmospheric correction 


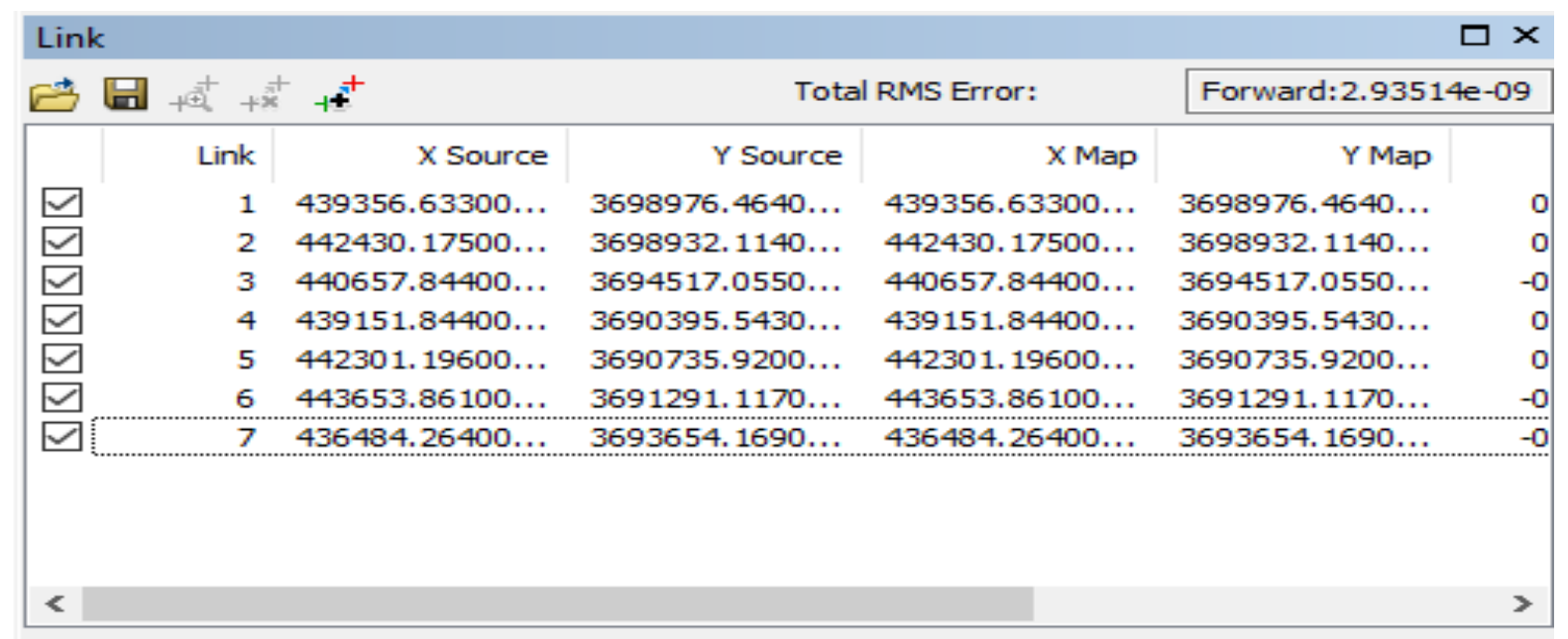

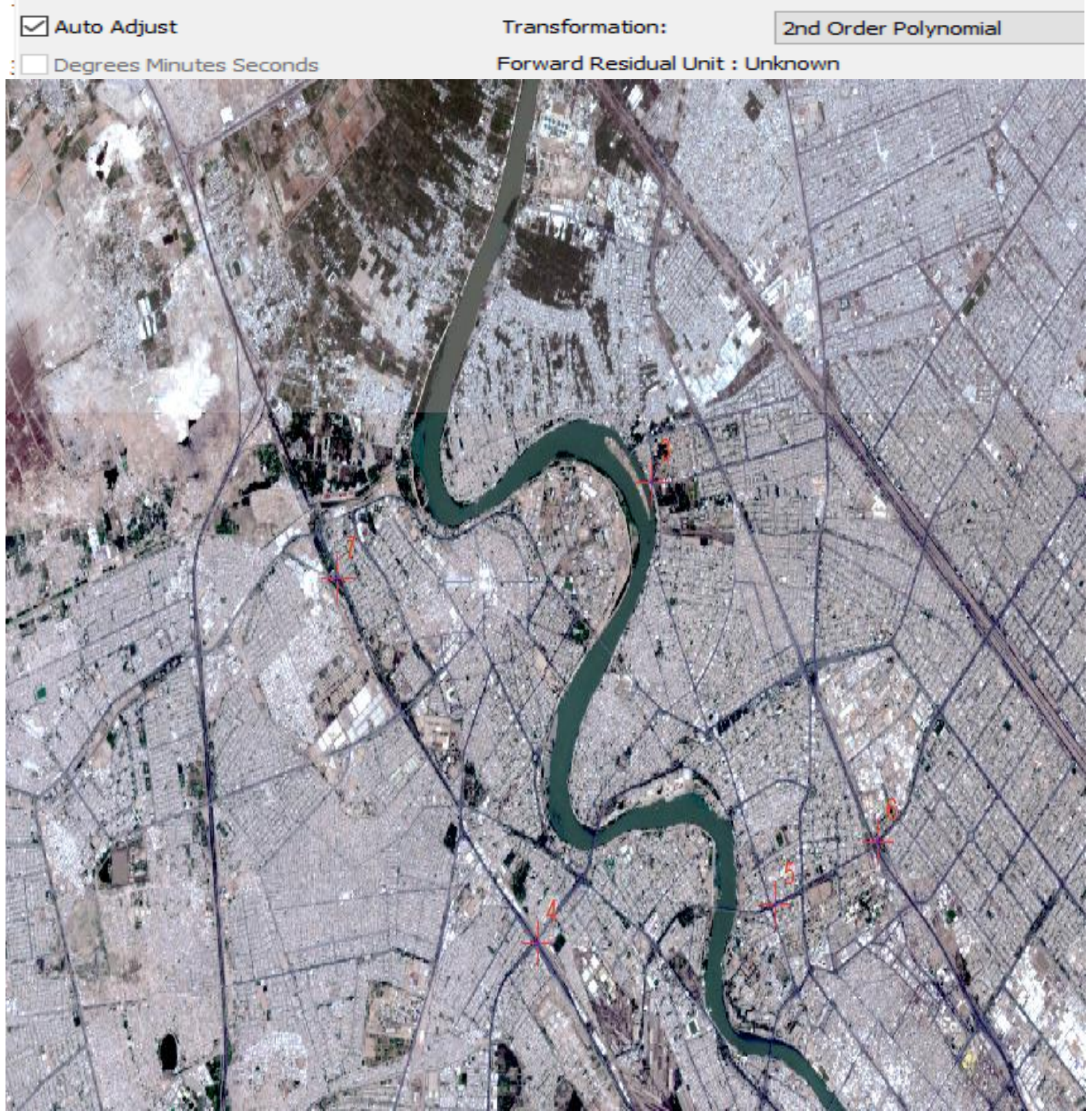

Figure 4. Processing of geometric correction for Sentinel-2 satellite image.

Mosaicking of Satellite Images. Image mosaicking is carried out based on a standard system of references/coordinates reported in meta-data. This system can be a real-world coordinate system (in other words, a projection of a map) or merely a grid defining pixel positions in an image. Whether 
there is a possibility of choosing between those two depends on the information contained in metadata, to a high level depending on the level of the processing of the original product as well [13]. In some cases, the coordinates coming with the images are inaccurate, even though they can mosaic the neighboring tiles. There is a possibility of mosaicking the adjacent tiles based on every tile's upperleft corner pixel location (provided in metadata).

Image sub-setting of the study area. The Baghdad City research area is located in central Iraq with the following geographical coordinates: Longitude $\left(44^{\circ} 12^{\prime} \mathrm{E}\right)$ to $\left(44^{\circ} 33^{\prime} \mathrm{E}\right)$ and Latitude $\left(33^{\circ} 9^{\prime} \mathrm{N}\right)$ to $\left(33^{\circ} 30^{\prime} \mathrm{N}\right)$. The image is sub-setting to the "square size" boundary of the study area geometry form [14]. 3551 columns and 3271 rows make up the clipped image. This procedure is done using the shapefile clipping data tools in ArcGIS 10.8 software, as shown in Figure 5.

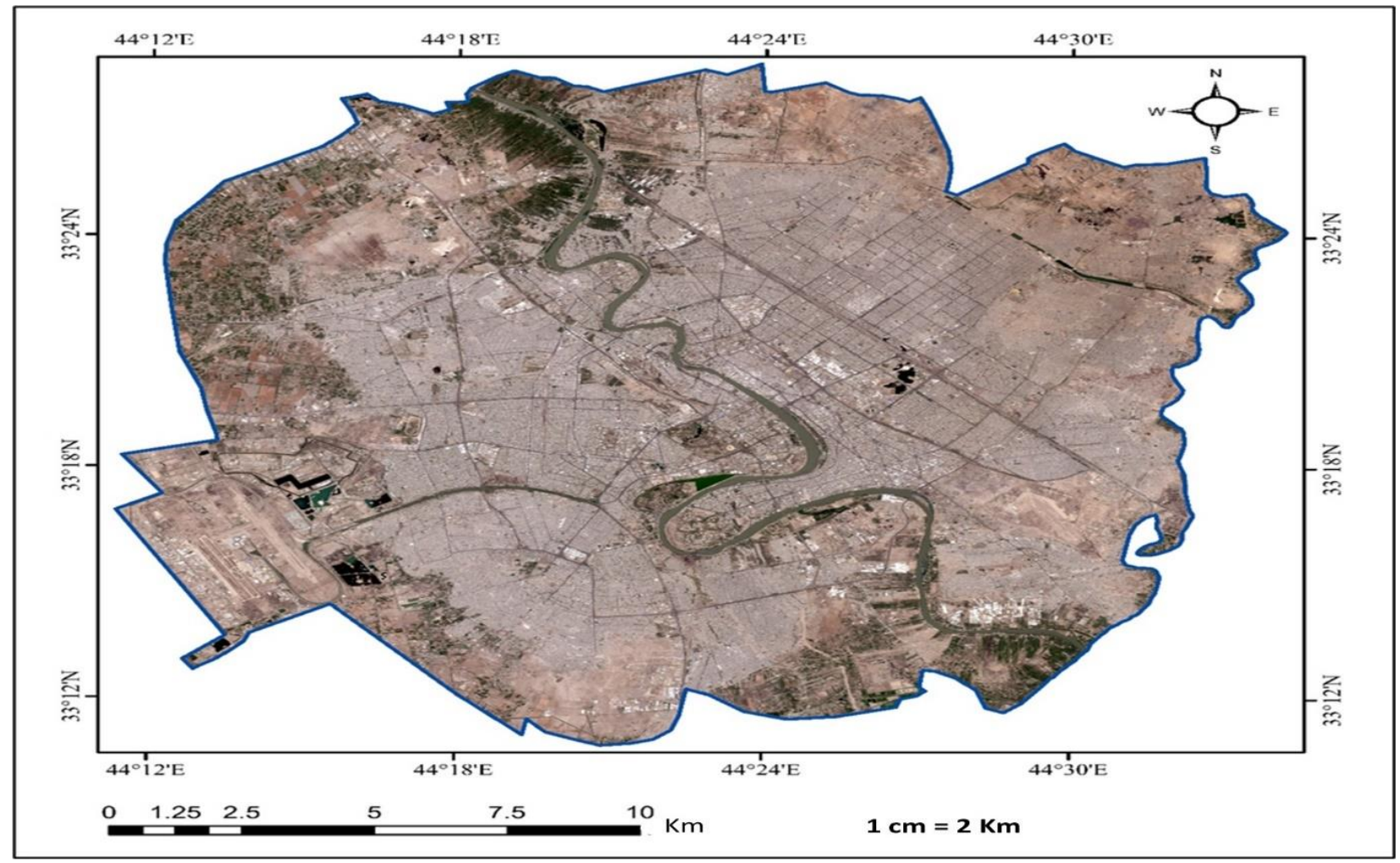

Figure 5. Final processing of satellite image for the study area.

\section{Results Analysis and Discussion}

The research field was divided into five major groups for different classifications centered on the most positioned ground truth sample positions within Baghdad city to obtain thematic maps of land use and land cover. They are five groups (vegetation area, asphalt road, soil area, water body, and built-up). Using Envi software, Training sites collected were chosen to be the region of interest (ROIs) polygons for each group and then used the following ROIs to classify the thematic map of LU/ LC for the study region. This stage was about selecting the study region's test sample; these samples are essential for evaluating each classifier's accuracy assessment checking the classification validation, supervising classification accuracy and producer accuracy, and user accuracy. Testing sites are typically samples of ground truth, and it the more comfortable to obtain pure ground truth samples from the field of the research area. The test sites for this analysis were obtained from office work. This section introduces and explains all the final effects of viewing and evaluating the outcome. The results of the classification are discussed initially, some many statistics by each class are measured, namely classification accuracy assessment. A method and results for accuracy assessment of (Urban area, Water bodies, Asphalt road, Vegetation, Soil) classification are analyzed using different classification such as Feature extraction and pixel-based for each SVM method MLC. 
After conducting supervised classifications techniques for the corrected images that represent the study area using the ENVI 5.3v software and to obtain accurate results, all classification results were converted into shapefiles to deal with the environment in the ArcGIS 10.8 and compute all areas of the chosen layers as shown in the section of supervised classifications results.

Classification Accuracy Assessment. The evaluation step of the satellite image classification involves the precision evaluation phase [1]. Accuracy assessment is an approximate quantitative analysis with a remotely sensed data set and is useful for testing the classification approach. Determining which errors may be involved is also essential. The precise evaluation of our classification methods is suggested as a matrix of confusion [1]. In this study, with the help of the collected GCPs from fieldwork (collected using GR5 DGPS), the accuracy assessment of the classifications performed by SVM and MLC was randomly selected to validate our classification results with our test sample.

Feature extraction Result. Using (SVM) algorithm and (MLC) algorithm, supervised classifications were performed for all corrected images representing the study area using ENVI 5.3v. Classification of results into the ArcGIS 10.8v environment and generation of thematic maps is categorized into five major categories and global standards, including vegetation, soil, asphalt roads, water bodies, and urban areas. Also, to recognize and differentiate the graded areas shown in Figures 6 and 7, each color was chosen to be similar to the class's natural color and to know the areas and percentages of the classes shown in Table 1.

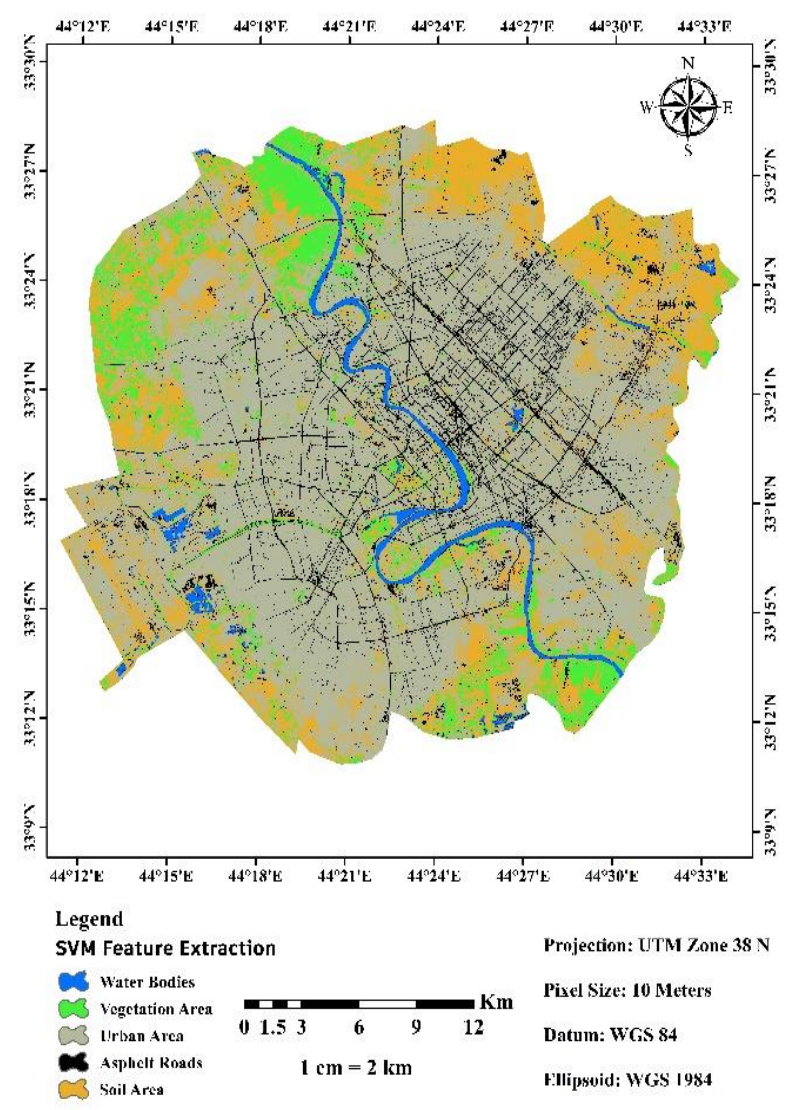

Figure 6. Supervised classification feature extraction map using (SVM).

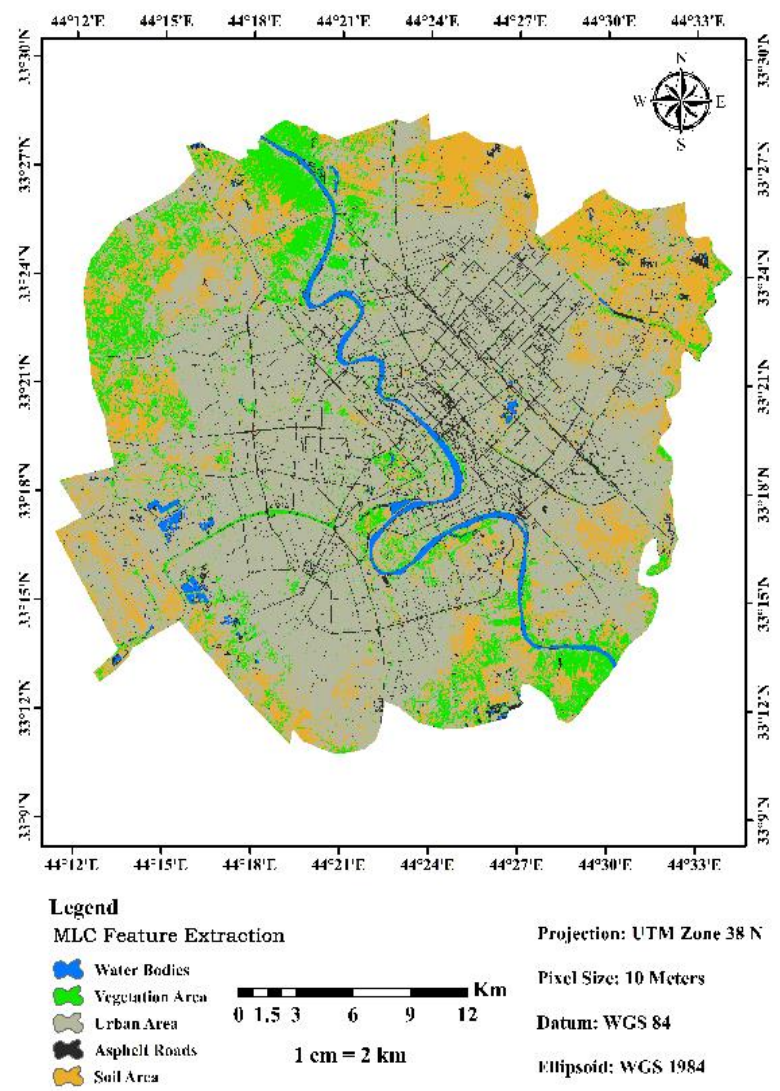

Figure 7. Supervised classification feature extraction map using (MLC). 
Table 1. Area and percentages of Feature extraction (SVM and MLC) classes for study area.

\begin{tabular}{|c|c|c|c|c|}
\hline \multirow{2}{*}{ Classes } & \multicolumn{2}{|c|}{ Feature extraction MLC } & \multicolumn{2}{c|}{ Feature extraction SVM } \\
\cline { 2 - 5 } & ${\text { Area, } \mathbf{~ k m}^{\mathbf{2}}}$ & Percentage, $\%$ & Area, $\mathbf{~ k m}^{\mathbf{2}}$ & Percentage, $\%$ \\
\hline Urban Area & 239.559 & 28.74 & 281.559 & 33.78 \\
\hline Soil Area & 124.057 & 14.89 & 101.057 & 12.13 \\
\hline Water Bodies & 61.651 & 7.40 & 62.651 & 7.52 \\
\hline Vegetation Area & 365.762 & 43.89 & 343.762 & 41.25 \\
\hline Asphalt Roads & 42.367 & 5.08 & 44.367 & 5.32 \\
\hline Total & 833.397 & 100 & 833.397 & 100 \\
\hline
\end{tabular}

Pixel Based Result. Using (SVM) algorithm and (MLC) algorithm, supervised classifications were performed for all corrected images representing the study area using ENVI 5.3v only. Classification of results into the ArcGIS 10.8v environment and generation of thematic maps, categorized into five major categories and according to global standards, including vegetation area, soil area, asphalt roads, bodies of water, and urban areas. Also, to recognize and differentiate the graded areas shown in Figures 8 and 9, each color was chosen to be similar to the class's natural color and to know the areas and percentages of the classes shown in Table 2.

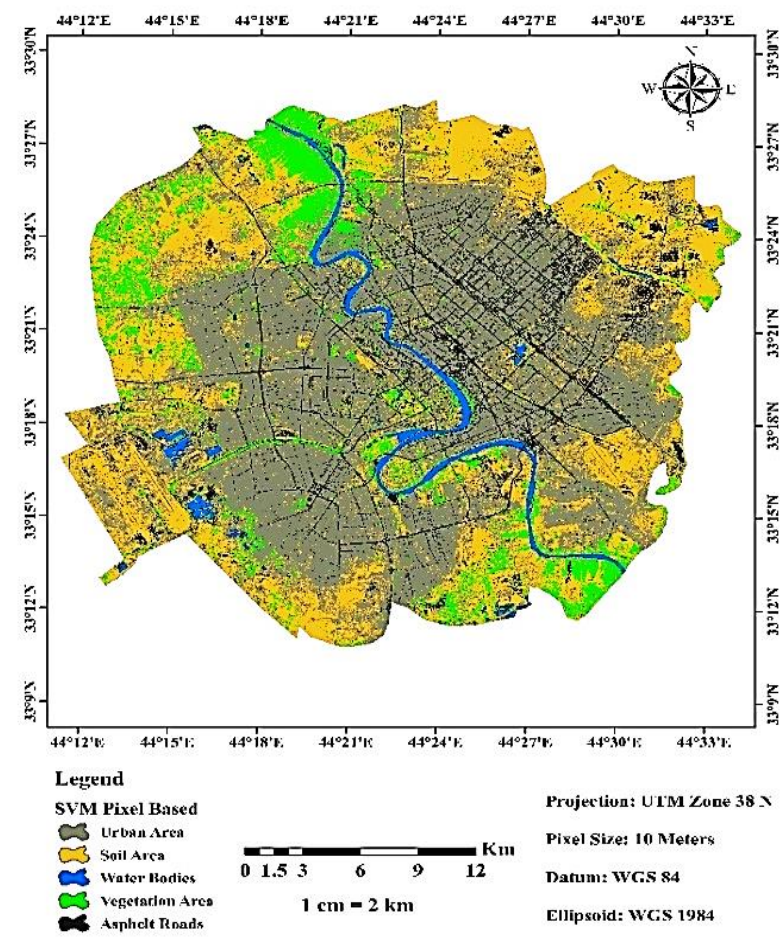

Figure 8. Supervised classification pixelbased map using (SVM).

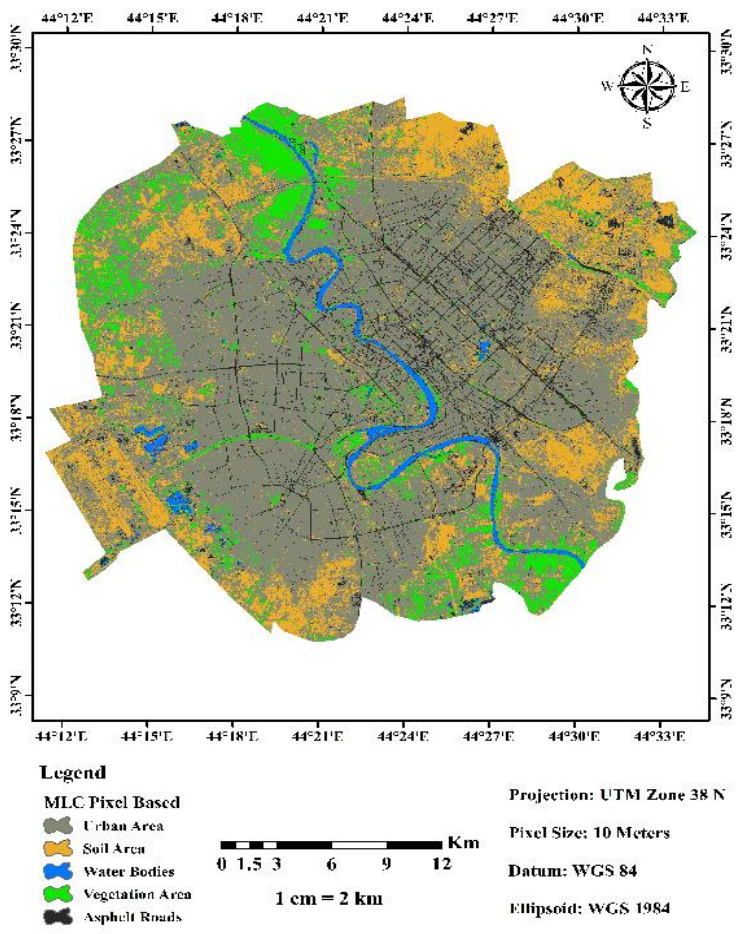

Figure 9. Supervised classification pixelbased map using (MLC).

Table 2. Area and percentages of pixel base (SVM and MLC) classes of the study area.

\begin{tabular}{|c|c|c|c|c|}
\hline \multirow{2}{*}{ Classes } & \multicolumn{2}{|c|}{ Pixel base MLC } & \multicolumn{2}{c|}{ Pixel base SVM } \\
\cline { 2 - 5 } & Area, $\mathbf{k m}^{\mathbf{2}}$ & Percentage, $\%$ & Area, $\mathbf{k m}^{\mathbf{2}}$ & Percentage, \% \\
\hline Urban Area & 241.559 & $28.98 \%$ & 261.559 & $31.38 \%$ \\
\hline Soil Area & 141.057 & $16.93 \%$ & 121.057 & $14.53 \%$ \\
\hline Water Bodies & 62.651 & $7.52 \%$ & 42.651 & $5.12 \%$ \\
\hline Vegetation Area & 355.762 & $42.69 \%$ & 373.762 & $44.85 \%$ \\
\hline Asphalt Roads & 32.367 & $3.88 \%$ & 34.367 & $4.12 \%$ \\
\hline Total & 833.397 & $100 \%$ & 833.397 & $100 \%$ \\
\hline
\end{tabular}


Comparison Analysis Results. After conducting all kinds of classifications, it compared the allclassification accuracy to know which technique has the highest accuracy and can perform this research for a LU/LC Table 3. Figures 10 and 11 show a comparison of the classifications method in the overall accuracy and Kappa index level.

Table 3. Comparison between all applied classifications.

\begin{tabular}{|c|c|c|c|}
\hline \multicolumn{4}{|c|}{ Feature extraction } \\
\hline No. & techniques & Overall accuracy, \% & Kappa Index \\
\hline 1 & SVM & $95 \%$ & 0.94 \\
\hline 2 & MLC & $92 \%$ & 0.90 \\
\hline \multicolumn{4}{|c|}{ Pixel Based } \\
\hline No. & techniques & Overall accuracy, \% & Kappa Index \\
\hline 1 & SVM & $88 \%$ & 0.84 \\
\hline 2 & MLC & $86 \%$ & 0.82 \\
\hline
\end{tabular}

Table 3 indicates the comparison between all applied classifications (Feature extraction and Pixelbased) in the overall accuracy and the level of Kappa index. It is clear from the table that (Feature extraction) is a superior method with the highest values of overall accuracy and Kappa index.

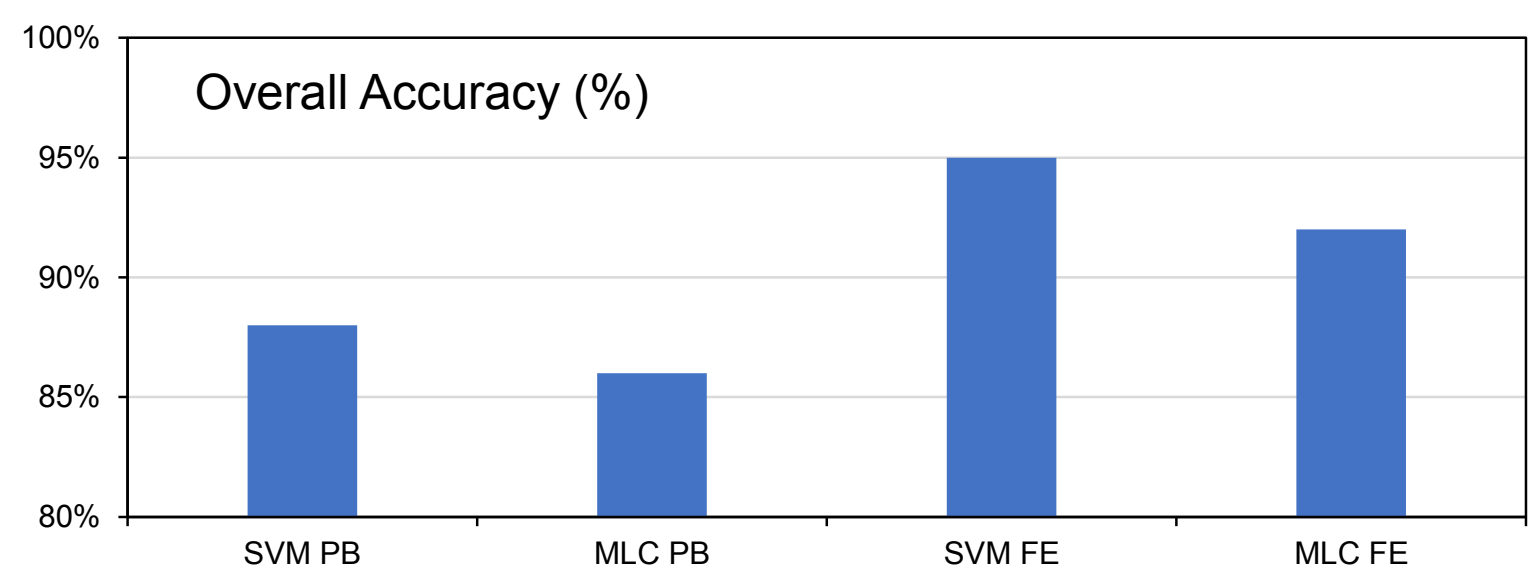

Figure 10. The overall accuracy of all classification

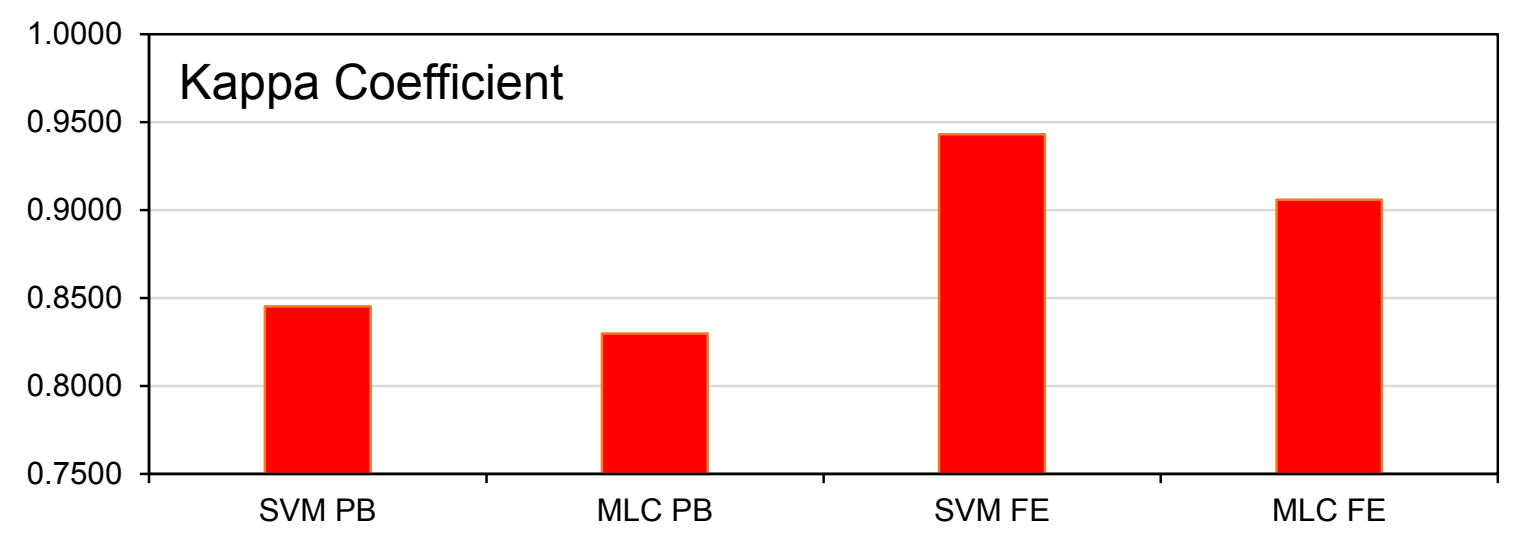

Figure 11. The kappa coefficient of all classification.

The effects of the kappa index and the two classification methods' overall accuracy are revealed in both Figures 10 and 11. The producer's precision, user accuracy, overall accuracy, and kappa index were computed in Tables 3 to compare the Pixel-based and Feature extraction classifications. The findings indicate better performance for Feature extraction classifiers. With $95 \%$ overall accuracy and kappa index of 0.94 for SVM classifier and 92\% overall accuracy and kappa index of 0.91 for MLC classifier, the Pixel-based classifier produces the most precise results with $88 \%$ overall accuracy and kappa index of 0.84 for SVM and $86 \%$ overall accuracy and kappa classifier. Due to their ability 
to effectively manage limited training data sets, Feature extraction objects are especially attractive in the remote sensing field, often achieving higher classification accuracy than conventional methods.

\section{Conclusions}

This study conducted a comparative analysis of Feature extraction and pixel-based image analysis performance to classify land use/land cover in Baghdad, Iraq, using Sentinel-2 image.

- The study results found that it is mighty and feasible for this analysis to use RS technology and GIS to detect the LU/LC.

- The Feature extraction method has the most reliable land use and landcover results by obtaining high accuracy results. By achieving general accuracy of $95 \%$ for SMV and $92 \%$ for MLC, the Support Vector Machine classifier has the most accurate land use and landcover performance.

- The SVM and MLC for pixel-based approaches seem to have an average precision of $88 \%$ and $86 \%$, respectively. The Support Vector Machine Classifier's Kappa index is also the highest total algorithm used for this analysis. Concerning the comparison of the overall accuracy and kappa index of the algorithms used, it is clear that the best method is the Feature extraction results.

- To remove the noise problem, segmentation has its unique superiority of tiny noise pixels, and the neighboring areas constitute several homogeneous objects in the segmentation process, and it is the objects that are categorized. To some extent, the signal-to-noise ratio in the image is more or less increased by segmentation.

\section{References}

[1] Abburu, S. and Golla, S.B., 2015. Satellite image classification methods and techniques: A review. International journal of computer applications, 119(8).

[2] Trock, C. F. S., 2018. Land cover classification of urban areas : A comparison of object-based and pixel-based approaches. M.Sc. Thesis, Aalborg University Copenhagen.

[3] Bukheet, Y.C., Al-Abudi, B.Q. and Mahdi, M.S., 2016. Land Cover Change Detection of Baghdad City Using Multi-Spectral Remote Sensing Imagery. Iraqi Journal of Science, pp.195214.

[4] Aggarwal, N., Srivastava, M. and Dutta, M., 2016. Comparative analysis of pixel-based and object-based classification of high resolution remote sensing images-A review. International Journal of Engineering Trends and Technology, 38(1), pp.5-11.

[5] Mather, P. and Tso, B., 2016. Classification methods for remotely sensed data. CRC press.

[6] Hay, G.J. and Castilla, G., 2006, July. Object-based image analysis: strengths, weaknesses, opportunities and threats (SWOT). In Proc. 1st Int. Conf. OBIA (pp. 4-5).

[7] Abbas, Z. and Jaber, H.S., 2020, March. Accuracy assessment of supervised classification methods for extraction land use maps using remote sensing and GIS techniques. In IOP Conference Series: Materials Science and Engineering (Vol. 745, No. 1, p. 012166). IOP Publishing.

[8] TAATI, A., SARMADIAN, F., MOUSAVI, A., POUR, C.T.H. and SHAHIR, A.H.E., 2015. Land use classification using support vector machine and maximum likelihood algorithms by Landsat 5 TM images. Walailak Journal of Science and Technology (WJST), 12(8), pp.681-687.

[9] Merzah, Z.F. and Jaber, H.S., 2020, March. Assessment of Atmospheric Correction Methods for Hyperspectral Remote Sensing Imagery Using Geospatial Techniques. In IOP Conference Series: Materials Science and Engineering (Vol. 745, No. 1, p. 012123). IOP Publishing.

[10] Changhui, Y., Shaohong, S., Jun, H. and Yaohua, Y., 2010, April. An object-based change 
detection approach using high-resolution remote sensing image and GIS data. In 2010 International Conference on Image Analysis and Signal Processing (pp. 565-569). IEEE.

[11] Miranda, E., Mutiara, A.B. and Wibowo, W.C., 2018, September. Classification of land cover from Sentinel-2 imagery using supervised classification technique (preliminary study). In 2018 International Conference on Information Management and Technology (ICIMTech) (pp. 69-74). IEEE.

[12] Oommen, T., Misra, D., Twarakavi, N.K., Prakash, A., Sahoo, B. and Bandopadhyay, S., 2008. An objective analysis of support vector machine based classification for remote sensing. Mathematical Geosciences, 40(4), pp.409-424.

[13]Blaschke, T., 2010. Object based image analysis for remote sensing. ISPRS journal of photogrammetry and remote sensing, 65(1), pp.2-16.

[14] Li, M., Zang, S., Zhang, B., Li, S. and Wu, C., 2014. A review of remote sensing image classification techniques: The role of spatio-contextual information. European Journal of Remote Sensing, 47(1), pp.389-411. 